Pacific Journal of Mathematics

INTEGRAL DOMAINS THAT LOSE IDEALS IN OVERRINGS

Henze and David C. Santa 


\title{
INTEGRAL DOMAINS THAT LOSE IDEALS IN OVERRINGS
}

\author{
William Heinzer and DAvid LANTZ
}

\begin{abstract}
We study domains with the property that, in any proper overring, some proper ideal extends to the unit ideal, and domains with the property that, in any proper overring, some proper ideal of a certain type (v-ideal, invertible ideal, or principal ideal) extends to the unit ideal. We characterize the Noetherian domains with these properties, and we show that even the strongest of these properties does not imply the QR-property.
\end{abstract}

0. Introduction. An integral domain $D$ with field of fractions $K$ is said to have the "QR-property" if every subring of $K$ that properly contains $D$ (i.e., every "proper overring" of $D$ ) is a ring of fractions of $D$ with respect to some multiplicatively closed set. Robert Gilmer has asked whether a sufficient condition for $D$ to have the QR-property is that, for every proper overring $E$ of $D$, some nonunit of $D$ is a unit in $E$. In the present paper we show that the answer is no, essentially because even if $D$ satisfies the latter condition, a ring of fractions over it need not satisfy it. Another way of phrasing the latter condition is: For every proper overring $E$ of $D$, there is a proper principal ideal of $D$ that "blows up", i.e., extends to the unit ideal, in $E$. In these terms, it is natural to extend the idea to classes of ideals other than the class of principal ideals; for instance, it is more likely that a maximal ideal will blow up than a principal ideal. Thus, we were led to the following definition:

Definition. We call an integral domain $D$ "plosive" if, in every proper overring $E$ of $D$, some proper ideal of $D$ extends to the unit ideal in $E$. Letting "blue" denote any of the adjectives "v", "invertible", or "principal", we call $D$ "blue-plosive" if, in every proper overring $E$ of $D$, some proper blue ideal of $D$ extends to the unit ideal of $E$.

In these terms, then, Gilmer's question was whether a principalplosive domain has the QR-property; and examples below show that this is not true and that a principal-plosive domain may have a ring of fractions that is not principal-plosive. 
Gilmer's question was asked independently on page 9 of [AA]. In response, Dobbs [Do] has shown that $D$ has the QR-property if and only if, for any overrings $R_{1}$ and $R_{2}$ of $D$ with $R_{1}$ properly contained in $R_{2}$, there are elements of $D$ that are units in $R_{2}$ but not in $R_{1}$.

Let us begin with some simple observations. First, since every maximal ideal in a domain is the contraction of a maximal ideal in the integral closure, a plosive domain is integrally closed. Next, clearly a principal-plosive domain is invertible-plosive, an invertible-plosive domain is v-plosive, and a v-plosive domain is plosive. Finally, if an ideal extends to the unit ideal in some overring, then so do all the ideals containing it and some finitely generated ideal contained in it. Thus, we have not defined "finitely generated-plosive" or "maximalplosive", for these concepts are equivalent to "plosive"; nor have we defined "t-plosive" or "v-finite-plosive", for they are equivalent to " $v$ plosive".

In $\S 1$ below we consider plosive domains. We note that a plosive domain with only finitely many maximal ideals is Bezout. We show that, for a domain $D$ that is the intersection of a locally finite family of quasilocal one-dimensional overrings (e.g., a Krull domain), $D$ is plosive if and only if it is a generalized Krull domain in which each prime of height one is an intersection of maximal ideals. We note as corollaries that any normal Noetherian Hilbert domain and any polynomial ring in two indeterminates over a normal Noetherian domain is plosive. But an example shows that a normal Hilbert domain that is not Noetherian need not be plosive.

In $\S 2$ we consider v-plosive, invertible-plosive, and principal-plosive domains. We show that a Prüfer v-multiplication domain of finite character is $v$-plosive if and only if maximal $t$-ideals are maximal ideals of the domain; and that a Prüfer domain is principal-plosive if and only if every proper finitely generated ideal is contained in the radical of a proper principal ideal. (By [P, Theorem 5], a domain has the QR-property if and only if it is a Prüfer domain in which the radical of every finitely generated ideal is the radical of a principal ideal.) We show that, for a domain that is the intersection of a locally finite family of quasilocal one-dimensional overrings, the properties "v-plosive" and "invertible-plosive" are equivalent to each other and to the condition that the domain is one-dimensional Prüfer with Noetherian spectrum.

Section 3 provides two examples of principal-plosive domains that do not have the QR-property. One is the intersection of a family of 
one-dimensional quasilocal overrings, and the other is the intersection of a locally finite family of rank two valuation domains.

All rings are commutative with unity, and subrings share the unity of the larger ring. The symbols " $<$ " and " $>$ " between sets denote proper containment. We call a family of domains with the same field of fractions a "locally finite" family if any nonzero element of the field of fractions is a nonunit in at most finitely many members of the family. Such a family has the same intersection as an "irredundant" subfamily, i.e., a subfamily $F$ such that, for every member of $F$, there is an element of the field of fractions that is not in that member but is in every other member of $F$. The property of a locally finite family that we use most frequently is that a ring of fractions of the intersection with respect to a multiplicatively closed subset is the intersection of the rings of fractions of the members of the family with respect to the same subset, and the family of localizations is still locally finite [Gi2, (43.5), page 527]. (The hypothesis that the members of the family are overrings of the intersection is not needed, but we will usually apply it in that case.) A consequence is that every prime in the intersection is a union of primes contracted from members of the family. We call a domain a "generalized Krull domain" if its localizations at all primes of height one are (rank one) valuation domains, these localizations form a locally finite family, and the domain is the intersection of these localizations [Gi2, page 524]. The "v-closure" $I_{v}$ of an ideal $I$ in a domain is the intersection of the principal fractional ideals containing $I$; if $I_{v}=I$, then $I$ is a " $v$-ideal". An ideal of the form $I_{v}$ for some finitely generated ideal $I$ is a "v-finite" ideal. The "t-closure" $I_{t}$ of an ideal $I$ is the union of the v-closures of the ideals generated by finite subsets of $I$; if $I_{t}=I$, then $I$ is a "t-ideal" [J, page 19]. Maximal t-ideals exist and are prime ideals, and a domain is the intersection of the localizations at its maximal t-ideals. A domain $D$ is a "Prüfer v-multiplication domain", abbreviated PVMD, if each finitely generated ideal is "t-invertible", i.e., if, for any finitely generated ideal $I,\left(I I^{-1}\right)_{t}=D$ [Gi2, page 427]. The localization of a PVMD at a maximal t-ideal is a valuation domain [Gr1, Theorem 5, page 715]. A PVMD for which the family of localizations at maximal t-ideals is locally finite is "of finite character". Thus, a generalized Krull domain is a PVMD of finite character. A "GCD-domain" is a domain in which every pair of elements has a greatest common divisor. For elements $a$ and $b$ of a GCD-domain $D$, with greatest common divisor $d,(a, b)_{v}=(d)$ [Au, page 330]; 
thus, a GCD-domain is a PVMD. In a GCD-domain, the concepts of $\mathrm{v}$-plosive, invertible-plosive, and principal-plosive domain agree.

1. Plosive domains. In trying to determine circumstances under which plosive domains have the QR-property, we asked whether a quasilocal plosive domain has to be a valuation domain and whether the plosive property localizes. The answer to the second question is negative, as we shall see in a moment; but the answer to the first is positive:

Proposition 1.1. A plosive domain with only finitely many maximal ideals is a Bezout domain.

Proof. Let $D$ be a plosive domain with only finitely many maximal ideals $M$, and for each $M$ pick a valuation overring of $D$ dominating $D_{M}$. Then no maximal ideal of $D$ extends to the unit ideal in the intersection $E$ of these valuation domains, so $D=E$. By $[\mathbf{K}$, Theorem 107, page 78], $D$ is a Bezout domain.

We next determine the Noetherian plosive domains; in fact, our result is somewhat more general. In the proof we make repeated use of the following: Let $M$ be a maximal ideal in the domain $D$ and $u$ be a nonzero element of the field of fractions of $D$. By $[K$, Theorem 67, page 40], if neither $u$ nor $u^{-1}$ is in $D_{M}$, then the kernel of the natural surjection $D_{M}[X] \rightarrow D_{M}[u]$ is contained in $M D_{M}[X]$; so $M$ does not extend to the unit ideal in $D[u]$.

THEOREM 1.2. Suppose the domain $D$ is the intersection of a locally finite family of quasilocal one-dimensional overrings. Then $D$ is plosive if and only if it is a generalized Krull domain in which each height one prime is an intersection of maximal ideals. In this case, if the family of overrings is irredundant, then each is a localization of $D$ and $a$ valuation domain.

Proof. We may assume that the family of overrings $\left\{V_{\alpha}\right\}$ is irredundant. Suppose first that $D$ is plosive. We show first that the centers $P_{\alpha}$ of the $V_{\alpha}$ on $D$ are not related by inclusion. Assume that, for different indices $\alpha$ and $\beta, P_{\beta} \subseteq P_{\alpha}$, and pick $u$ in $\left(\bigcap_{\gamma \neq \alpha} V_{\gamma}\right)-V_{\alpha}$. Suppose that, for all maximal ideals $M$ containing $P_{\alpha}, u^{-1} \notin D_{M}$. Then we claim no maximal ideal of $D$ extends to the unit ideal in $D[u]$, which is a contradiction. For, if the maximal ideal $M$ contains 
$P_{\alpha}$, then neither $u$ nor $u^{-1}$ is in $D_{M}$; while if $M$ does not contain $P_{\alpha}$, then $D_{M}$ is an intersection of some of the $V_{\gamma}$ not including $V_{\alpha}$, so $u$ is in $D_{M}$. In either case $M$ does not extend to the unit ideal in $D[u]$. Thus, $u^{-1} \in D_{M}$ for some maximal ideal $M$ containing $P_{\alpha}$, and hence $u^{-1} \in V_{\alpha}$; and since $u \notin V_{\alpha}, u^{-1}$ is in the maximal ideal of $V_{\alpha}$. Take a nonzero element $a$ of $P_{\beta}$, and use the fact that $V_{\alpha}$ is one-dimensional quasilocal to find a power of $u^{-1}$ sufficiently large that $u^{-n} V_{\alpha}<a V_{\alpha}$. Then $t=a u^{n} \notin V_{\alpha}$, but $t$ is in the maximal ideal of $V_{\beta}$. The argument above shows that $t^{-1} \in D_{M}$ for some maximal ideal $M$ containing $P_{\alpha}$. But then $t^{-1}$ is also in the localization of $D$ at $P_{\beta}$ and hence in $V_{\beta}$, contradicting the fact that $t$ is in the maximal ideal of $V_{\beta}$.

It follows from the above and the fact that passage to a ring of fractions distributes over a locally finite intersection that the domains $V_{\alpha}$ are the localizations of $D$ at its height one primes; and so every element of $D$ is in only finitely many height one primes. It remains to show that the height one primes of $D$ are intersections of maximal ideals and that the localizations at these primes are valuation domains. For the former, assume there is a height one prime $P$ of $D$ that is properly contained in the intersection $I$ of the maximal ideals that contain it. Take a nonzero element $b$ of $P$, and choose an element $a$ in $I$ and in all the (finitely many, by the local finiteness) height one primes containing $b$ other than $P$, but not in $P$. Replace $a$ by a power, if necessary, so that, for each minimal prime $Q$ of the ideal $(a, b), b \notin a D_{Q}$. (It suffices to find a power of $a$ that does this in each of the $D_{Q}$ for $Q$ one of the finitely many height one primes containing a.) We claim that no maximal ideal $M$ of $D$ extends to the unit ideal of $D[a / b]$, a contradiction since $a$ is not in $P$ and hence is not a multiple of $b$. If $P \subseteq M$, then $a / b \notin D_{P}$, so $a / b \notin D_{M}$; and since $M$ contains $(a, b)$ and hence one of its minimal primes $Q$, and $b / a \notin D_{Q}$, we also have $b / a \notin D_{M}$. If $P \nsubseteq M$, then $a / b \in \bigcap\left\{D_{Q}: \operatorname{ht}(Q)=1, Q \subseteq M\right\}=D_{M}$. In either case $M$ does not extend to the unit ideal in $D[a / b]$.

Finally, we must show that a localization of $D$ at a height one prime $P$ is a valuation domain. Suppose not; then there is a valuation domain $W$ properly dominating $D_{P}$. Let $E$ denote the intersection of $W$ and the domains $V_{\alpha}$ other than $D_{P}$. Then $E>D$, for otherwise the locally finite family $\{W\} \cup\left\{V_{\alpha}: V_{\alpha} \neq D_{P}\right\}$ has an irredundant subfamily intersecting in $D$; one must be $W$, and we saw in the first paragraph of the proof that this implies $W$ is the localization of $D$ at 
its center, i.e., $W=D_{P}$. So take an element $u$ of $E-D$; we contend no maximal ideal $M$ of $D$ extends to the unit ideal in $D[u]$, a contradiction: If $P \nsubseteq M$, then $u \in \bigcap\left\{D_{Q}: \mathrm{ht}(Q)=1, Q \subseteq M\right\}=D_{M}$. If $P \subseteq M$, then neither $u$ nor $u^{-1}$ is in $D_{M}$. For, if $u \in D_{M}$, then $u \in D_{P}$, contrary to our choice of $u$; while if $u^{-1} \in D_{M}$, then $u^{-1} \in D_{P} ;$ it is a nonunit there and also in $W$, but $u \in W$.

For the converse, suppose $D$ is a generalized Krull domain in which each height one prime is an intersection of maximal ideals. To show $D$ is plosive, it suffices to take an element $u$ of the field of fractions of $D$ not in $D$ and show that some maximal ideal of $D$ extends to the unit ideal in $D[u]$. The hypotheses guarantee that, for some height one prime $P$ of $D, u \notin D_{P}$, and $D_{P}$ is a rank one valuation domain. Now if $P D[u] \cap D=P$, then $P D[u]$ is contracted from $D[u]_{D-P} \supseteq D_{P}[u]$; but the last ring is a field. So at least one of the maximal ideals $M$ intersecting in $P$ is such that $M D[u] \cap D>M$, i.e., $M$ extends to the unit ideal in $D[u]$.

COROLlary 1.3. A Noetherian domain is plosive if and only if it is an integrally closed domain in which each height one prime ideal is an intersection of maximal ideals.

Thus, the property of being plosive does not pass to rings of fractions: A polynomial ring in two indeterminates over a field has the property, but its localization at a maximal ideal does not. In fact:

Corollary 1.4. A normal Noetherian Hilbert domain is plosive.

But a normal Hilbert domain that is not Noetherian need not be plosive: Let $k$ be a field and $x$ and $t$ be indeterminates over it. The domain $D=k+x k(t)[x]$ is integrally closed, and the contraction map $\operatorname{Spec}(k(t)[x]) \rightarrow \operatorname{Spec}(D)$ is an isomorphism. (Cf. [BR, MZ].) Thus, $D$ is Hilbert, but it is not plosive.

On the other hand, a Noetherian plosive domain need not be Hilbert. In fact, if $D$ is a normal Noetherian domain and $x$ and $y$ are indeterminates, then $D[x, y]$ is plosive. (For, let $P$ be a height one prime in $D[x, y]$. If $P \cap D[x] \neq(0)$, then $P$ is generated by this intersection, and forming the factor ring with respect to $P$ yields a polynomial ring in the image of $y$ over a domain, which has zero Jacobson radical. If $P \cap D[x]=(0)$, then the factor ring with respect to $P$ is a domain extension of $D[x]$ finitely generated as an algebra, and 
since $D[x]$ has zero Jacobson radical, so does the factor ring [AM, Exercise 22, page 70; Gi1, Corollary 1, page 282]. Thus in either case $P$ is an intersection of maximal ideals.) But when $D$ is not Hilbert, then neither is $D[x, y]$. In particular, if $V$ is a discrete rank one valuation domain, then $V$ is plosive, $V[x]$ is not plosive, and $V[x, y]$ is plosive. But under suitable hypotheses this is the only case of bad behavior of the plosive property in finitely generated normal domain extensions. The "dimension formula" of the next proposition appears in [M, Theorem 15.5, pages 118-119] and [ZS, Appendix 1, page 326].

Proposition 1.5. Let $D$ be a Noetherian plosive domain that satisfies the dimension formula, and let $E$ be a normal domain containing $D$ and finitely generated as an algebra over $D$. Suppose that either $E$ is algebraic over $D$ or $D$ is not one-dimensional semilocal. Then $E$ is also plosive.

Proof. Let $Q$ be a height one prime in $E$. If the height of the prime $P=Q \cap D$ is zero, then $E$ is not algebraic over $D$, so $D$ has infinitely many height one primes (or $D$ is a field). Since each height one prime is an intersection of maximal ideals, $D$ has zero Jacobson radical. Now, $E / Q$ is a finitely generated extension of $D$, so $E / Q$ also has zero Jacobson radical [AM, Exercise 22, page 70; Gi1, Corollary 1, page 282]. If $P$ has height one, then $P$ is an intersection of maximal ideals, so $D / P$ has zero Jacobson radical and hence so does $E / Q$. If $P$ has height greater than one, then the dimension formula yields that the transcendence degree of $E / Q$ over $D / P$ is positive, so $E / Q$ is finitely generated over a polynomial ring over $D / P$, and hence again $E / Q$ has zero Jacobson radical. Thus, in each case, $Q$ is an intersection of maximal ideals.

We do not know whether the dimension formula hypothesis in this proposition is necessary. Also, we do not know whether it is possible, in a normal Noetherian domain, for only a finite number of height one primes to fail to be intersections of maximal ideals, unless that number is zero.

2. V-, invertible-, and principal-plosive domains. In order to have plenty of $\mathrm{v}$-ideals, the results of this section often include the hypothesis of PVMD, so that the v-finite ideals form a group under v-multiplication: $I \times_{v} J=(I J)_{v}$. Given a PVMD $D$ and a valuation overring $W$, it is tempting to believe that the group of v-finite ideals is embedded in the value group of $W$, by taking the minimum 
value of an element of the ideal as the value of the ideal. This is true, of course, of the subgroup of principal fractional ideals. The trouble is that this mapping need not be a group homomorphism. For instance, let $k$ be a field and $x$ and $y$ be indeterminates, and consider the domain $D=k\left[x^{2}, x y, y^{2}\right]$ (which can also be realized as $\left.k[u, v, w] /\left(u w-v^{2}\right)\right)$. Let $G$ denote the product of two copies of the integers, lexicographically ordered left to right, and define the valuation overring $W$ of $D$ by assigning to $x$ and $y$ (in the extension $k[x, y]$ of $D)$ the values $(1,0)$ and $(0,1)$ respectively. Then the inverse of the ideal $I=\left(x^{2}, x y\right)$ of $D$ is $(1, y / x)$ (since the ideal $\left(x^{2}, x y, y^{2}\right)$ has v-closure $\left.D\right)$, so the value $(-1,1)$ of the inverse of $I$ is not the negative $(0,-1)$ of the value of $I$. When a valuation overring is a localization of a domain, however, the value mapping on $\mathrm{v}$-finite ideals of the domain is an ordered semigroup homomorphism [HO3, page 859].

In preparation for the first result of the section, we note the following. Let $D$ be a PVMD of finite character. Then the representation of $D$ as the intersection of its localizations at maximal t-ideals is already irredundant. For, suppose $P$ is a maximal t-ideal, and let $E$ be the intersection of the localizations $D_{Q}$ of $D$ at all the maximal t-ideals $Q$ different from $P$. If $E=D$, then $P D_{P}$ is the union of primes contracted from rings of fractions of the domains $D_{Q}$. But $D_{P}$ is a valuation domain, so these primes form a chain; and a nonzero element of $P D_{P}$ is a unit in all but finitely many of the $D_{Q}$, so the members of the chain cannot all be proper. Since $P$ is not contained in any $Q$, this is a contradiction. This argument shows more in the case that $D$ is v-plosive: Since $P$ is the only maximal t-ideal that can extend to the unit ideal in the intersection $E$, there is a finitely generated ideal of $D$ that is contained in $P$ but not in any other maximal t-ideal.

Proposition 2.1. A Prüfer v-multiplication domain in which each maximal $t$-ideal is a maximal ideal is v-plosive. If a PVMD is of finite character and $v$-plosive, then every maximal $t$-ideal is maximal.

Proof. Let $D$ be a PVMD in which all maximal t-ideals are maximal ideals, and let $E$ be an overring of $D$. If no maximal t-ideal $P$ of $D$ extends to the unit ideal in $E$, then for each $P$ there is a maximal ideal $N(P)$ of $E$ lying over $P$; and since $E_{N(P)}$ dominates the valuation domain $D_{P}$, they are equal. Thus, $E \subseteq \bigcap_{P} E_{N(P)}=$ $\bigcap_{P} D_{P}=D$. So $D$ is v-plosive. 
Conversely suppose $D$ is a v-plosive PVMD of finite character, and let $P$ be a maximal t-ideal. If $P$ is not maximal, it is properly contained in a maximal ideal $M$, which is a union of primes contained in maximal t-ideals. One of these, say $Q$, is not contained in $P$. Take a valuation overring $W$ of $D$ with primes contracting to $Q$ and $M$. Since no maximal t-ideal of $D$ extends to the unit ideal in $E=$ $W \cap \bigcap_{N} D_{N}$, where $N$ varies over the maximal t-ideals not contained in $M$, we have $E=D$. Localizing the (locally finite) expression for $E$ at $P$, we see that $P D_{P}$ is a union of primes, one of which, say $P_{1}$, is contracted from $W$, while the rest are contracted from localizations of the domains $D_{N}$ with $N \nsubseteq M$. Let $I$ be a finitely generated ideal contained in $P$ but not in any other maximal t-ideal. Since $D_{P}$ is a valuation domain, $I D_{P}$ is principal, and no prime contracted from a localization of a $D_{N}$ contains its generator. Moreover, since $P_{1} \subseteq P D_{P}$, the prime in $W$ from which $P_{1}$ is contracted does not contain the one from which $Q$ was contracted; so the latter contains the former. But then $P_{1} \cap D \subseteq Q$, and since $Q$ is contained in a maximal t-ideal other than $P, I \nsubseteq Q$, so $I D_{P} \nsubseteq P_{1}$. Since none of the primes contracted from $W$ or the domains $D_{N}$ contain the generator of $I D_{P}$, their union is not $P D_{P}$, a contradiction.

Example 3.2 below shows that a PVMD of finite character in which each maximal $t$-ideal is a maximal ideal need not be a Prüfer domain. But Theorem 2.4 shows that a PVMD of finite character in which every maximal t-ideal is a height one maximal ideal is Prüfer.

The following corollary is immediate from Proposition 2.1.

COROLlaRY 2.2. A GCD-domain in which each maximal t-ideal is a maximal ideal is principal-plosive. If the GCD-domain is of finite character, then the converse holds.

Let $D$ be a Prüfer domain. Then for any element $u$ of the field of fractions of $D$, the denominator ideal of $u, I=D: D u=\{a \in D$ : $a u \in D\}$, is a finitely generated ideal of $D$ (since $D$ is coherent) and hence is invertible. Now

$$
D[u]=\bigcap\left\{D_{P}: P \in \operatorname{Spec}(D), u \in D_{P}\right\},
$$

since any overring of $D$ is flat [LM, Proposition 4.14, page 91, and Theorem 6.10, page 132]; so for any maximal ideal $M$ of $D[u]$, $I D[u]_{M}=(D: u) D_{M \cap D}=D_{M \cap D}: u=D_{M \cap D}=D[u]_{M}$, and hence $I$ extends to the unit ideal in $D[u]$. Thus, a Prüfer domain is invertibleplosive. (In fact, $D[u]$ is just the ideal transform $\bigcup_{n} I^{-n}$ of $I$.) 
Proposition 2.3. A Prüfer domain is principal-plosive if and only if each proper finitely generated ideal is contained in the radical of a proper principal ideal.

Proof. Let $D$ be a principal-plosive Prüfer domain, and let $I$ be a proper finitely generated ideal. Then the ideal transform $E$ of $I$ is a proper overring of $D$, so there is a nonunit $b$ of $D$ that is a unit in $E$. That means $b^{-1} I^{n} \subseteq D$ for some $n$, and so $I$ has a power contained in $b D$.

Conversely let $D$ be a Prüfer domain in which every proper finitely generated ideal is contained in the radical of a proper principal ideal, and let $u$ be in the field of fractions of $D$ but not in $D$. Then $D[u]$ is the ideal transform of the proper finitely generated ideal $I=D:_{D} u$, and in this ideal transform the generator of the proper principal ideal that contains a power of $I$ becomes a unit.

THEOREM 2.4. Let $D$ be a domain that is the intersection of a locally finite family of quasilocal one-dimensional overrings. Then the following conditions are equivalent:

(1) $D$ is v-plosive.

(2) $D$ is invertible-plosive.

(3) $D$ is a one-dimensional Prüfer domain with Noetherian spectrum. When these conditions hold, $D$ is principal-plosive if and only if each maximal ideal is the radical of a principal ideal.

Proof. We have seen that $(3) \Rightarrow(2)$, and $(2) \Rightarrow(1)$ is clear, so suppose $D$ is v-plosive. By Theorem 1.2, $D$ is a generalized Krull domain, so it is enough to show that each maximal ideal of $D$ has height one. Assume the maximal ideal $M$ has height greater than one. Since $M$ is the union of height one primes, $D_{M}$ is not a valuation domain. So there is an element $u$ of the field of fractions of $D$ for which neither $u$ nor $u^{-1}$ is in $D_{M}$; and multiplying by an element of $D-M$, we may assume that $u$ is in every $D_{Q}$ for $Q$ a height one prime not contained in $M$. No height one prime in $D$ extends to the unit ideal in $D[u]$; and every proper v-ideal in the generalized Krull domain $D$ is contained in some height one prime, so no proper v-ideal extends to the unit ideal, a contradiction.

The last assertion follows from (3) and Proposition 2.3.

We would like to remove some of the adjectives in the hypothesis that the domain $D$ is the intersection of a locally finite family 
of quasilocal one-dimensional overrings (though Example 3.1 shows that some such hypothesis is necessary). Removing the word "onedimensional" but adding "valuation", we see that, for a domain $D$ that is the intersection of an irredundant locally finite family of valuation domains $V_{\alpha}$, a localization of $D$ at a height one prime is a valuation domain, a localization of one of the $V_{\alpha}$ (since a quasilocal domain that is an intersection of finitely many valuation domains is equal to one of them). If we also assume that $D$ is invertible-plosive, then each of the centers of the $V_{\alpha}$ on $D$ contains an invertible ideal contained in no other such center, and we can almost repeat the second part of the proof of Proposition 2.1 to see that each such center is maximal. Removing the words "locally finite" and "one-dimensional" but adding "valuation" and "localization at a maximal ideal" allows us to mimic the first part of the proof of Proposition 2.1.

Proposition 2.5. Suppose a domain $D$ has a family of maximal ideals $P$ for which (1) each $P$ is the radical of an invertible (respectively principal) ideal, (2) each $D_{P}$ is a valuation domain, and (3) $D=$ $\bigcap_{P} D_{P}$. Then $D$ is invertible-plosive (respectively principal-plosive).

Proof. Let $E$ be a proper overring of $D$. If none of the maximal ideals $P$ in the family of the hypothesis extend to the unit ideal of $E$, then for each $P$ there is a maximal ideal $N(P)$ of $E$ lying over $P$. Since $E_{N(P)}$ dominates the valuation domain $D_{P}$, they are equal; so $E \subseteq \bigcap_{P} E_{N(P)}=\bigcap_{P} D_{P}=D$, a contradiction. Thus one of the maximal ideals $P$ in the family extends to the unit ideal in $E$, and hence so does some finitely generated ideal $I$ contained in $P$, and hence so does the power of $I$ contained in the invertible (respectively principal) ideal $J$ with radical $P$, and hence so does $J$.

In view of Theorem 2.4, we wonder whether a v-plosive domain is always invertible-plosive. We do not know this is true even in every PVMD of finite character, but it is true if the PVMD also has Noetherian spectrum. Recall that a domain $D$ is called "quasicoherent" if the inverse of each finitely generated nonzero fractional ideal of $D$ is again finitely generated.

Proposition 2.6. Let $D$ be a v-plosive Prüfer v-multiplication domain of finite character. Suppose that either (a) $D$ is quasicoherent and no maximal t-ideal is the union of the prime t-ideals properly contained within it, or (b) $D$ has Noetherian spectrum. Then $D$ is invertible-plosive. 
Proof. Let $P$ be a maximal t-ideal of $D$. We want to show that there is a finitely generated ideal $I$ with radical $P$. In case (b) such an $I$ clearly exists. In case (a) we note that $P$ contains a finitely generated ideal $J$ that is not contained in any other maximal t-ideal $Q$ (since it extends to the unit ideal in $\bigcap_{Q} D_{Q}$ ). The primes contained in $P$ are totally ordered by inclusion (since $D_{P}$ is a valuation domain), so the hypothesis on prime t-ideals assures that we can add a generator to $J$ so that $J$ is not contained in any prime t-ideal properly contained in $P$. Let $I$ denote the v-closure of $J$; since $P$ is a t-ideal, $I \subseteq P$. By quasicoherence $I$ is finitely generated, and $I$ has radical $P$ because minimal primes of a t-ideal are themselves t-ideals $[\mathbf{H H}$, Proposition 1.1(5), page 38; J, Théorème 9, page 30 ].

Since $I$ extends to the unit ideal in any extension of $D$ in which $P$ does so, it is enough to show that $I$ is invertible, i.e., that its localization at each maximal ideal is principal. At the maximal ideals not containing $I$ this is clear, so suppose $I$ is contained in the maximal ideal $M$. Then $P \subseteq M$ and so by Proposition $2.1 P=M$. Since $D_{P}$ is a valuation domain, $I D_{P}$ is principal.

It would be interesting to know whether the "locally finite" hypothesis is necessary in the above results. In this connection, we pose three questions, which by Theorem 2.4 have positive answers when the spectrum is Noetherian: (1) Must a one-dimensional, invertible-plosive domain be Prüfer? (2) Must a one-dimensional, principal-plosive Prüfer domain have the QR-property? (3) More generally, must a one-dimensional, principal-plosive domain have the QR-property?

3. Examples. In this section we provide two examples of principalplosive domains that do not have the QR-property. The first has already appeared in the literature in a different context; we recall its essential features.

ExAmple 3.1. The domain $D$ in [H2] is the intersection of a family (not locally finite) of quasilocal one-dimensional overrings and is principal-plosive, but it is not a Prüfer domain. This $D$ was constructed as follows: Let $k$ be a field and $y, z, x_{1}, x_{2}, \ldots$ be indeterminates. The valuation domain $k\left(z, x_{1}, x_{2}, \ldots\right)[y]_{(y)}$ has the form $k\left(z, x_{1}, x_{2}, \ldots\right)+M$ where $M$ is its maximal ideal; set $V \doteq$ $k\left(x_{1}, x_{2}, \ldots\right)+M, K_{n}=k\left(y, z, x_{1}, x_{2}, \ldots, x_{n}\right)$, and $V_{n}=V \cap K_{n}$. For each $n$ starting with $D_{1}=V_{1}$, let $R_{n+1}$ be the Kronecker function ring of $D_{n}$ with respect to the element $r=\left(1+y x_{n+1}\right) / y$ and $D_{n+1}=R_{n+1} \cap V_{n+1}$. Then the desired $D$ is the union of all the $D_{n}$. 
Since the localization $V$ of $D$ is not a valuation domain (and hence is not plosive, by Proposition 1.1), $D$ is not Prüfer and hence not QR; so it remains to show that it is principal-plosive. For this it suffices to take any $u$ in the field of fractions $K$ of $D$, not in $D$, and show there is a nonunit in $D$ that is a unit in $D[u]$.

For some $n, u \in K_{n-1}-D_{n-1}$ (note that $K_{i}$ is the field of fractions of $D_{i}$ ), and hence $u \in K_{n}-R_{n}$ since $R_{n}$ is a Kronecker function ring of $D_{n-1}$. Since $R_{n}$ is Bezout, $R_{n}[u]$ is a localization of $R_{n}$ and hence also of $D_{n}$ (since $R_{n}=D_{n}[1 / s]$ where $s=$ $\left.y /\left(1+y x_{n}\right)\right)$. Let $a \in D_{n}$ be a nonunit of $R_{n}$ that is a unit in $R_{n}[u]$, and pick $m$ so that $s^{m} V_{n} \subseteq a V_{n}$. (Such an $m$ exists because $V_{n}$ is one-dimensional quasilocal.) Then since $V_{n}=\left(D_{n}\right)_{P}$ where $P$ is the center of $V_{n}$ on $D_{n},\left(a R_{n} \cap D_{n}\right)\left(D_{n}\right)_{P} \supseteq\left(a D_{n}: s^{m}\right) V_{n}=a V_{n}: s^{m} V_{n}=$ $\left(D_{n}\right)_{P}$, so $a R_{n} \cap D_{n} \nsubseteq P$. Since $a V_{n} \cap D_{n}$ is $P$-primary, the ideals $a R_{n} \cap D_{n}, a V_{n} \cap D_{n}$ are relatively prime, so their intersection is their product: $a D_{n}=a D_{n} \cap D_{n}=a\left(R_{n} \cap V_{n}\right) \cap D_{n}=\left(a R_{n} \cap a V_{n}\right) \cap D_{n}=$ $\left(a R_{n} \cap D_{n}\right) \cap\left(a V_{n} \cap D_{n}\right)=\left(a R_{n} \cap D_{n}\right)\left(a V_{n} \cap D_{n}\right)$. Thus $a R_{n} \cap D_{n}$ is invertible, hence finitely generated, hence its extension to the Kronecker function ring $R_{n+1}$ of $D_{n}$ is principal, generated by $b$, say. Since $a R_{n} \neq R_{n}, b$ is not a unit of $R_{n+1}$. Moreover, since $R_{n+1}$ is the Kronecker function ring of $D_{n}$ with respect to $r=\left(1+y x_{n+1}\right) / y$ and hence also with respect to $r^{-1} \in \max (V)$, we can choose $b$ to be of the form $a_{0}+a_{1} r^{-1}+\cdots+a_{k} r^{-k}$ where $\left(a_{0}, \ldots, a_{k}\right) D_{n}=a R_{n} \cap D_{n}$ and $a_{0} \notin \max (V)$. Then $b \in D_{n+1}$ but $b \notin \max (V)$, so $b$ is a unit in $V_{n+1}$ and hence in $V_{n+1}[u]$. To see $b$ is a unit in $R_{n+1}[u]$ (a localization of $R_{n+1}$ and hence integrally closed), it is enough to note that, if $W$ is a valuation overring of $R_{n+1}[u]$, then either $W$ is centered on $P$ in $D_{n}$, in which case $b$ must be a unit in $W$ since it has multiples (in $a R_{n} \cap D_{n}$ ) that are units in $W$; or $W$ is centered on another prime in $D_{n}$, in which case $R_{n}[u]=D_{n}[1 / s][u] \subseteq W$, so $a$ and hence $b$ are units in $W$.

Now to show $b$ is a unit in $D_{n+1}[u]$, it is enough to show it is a unit in the integral closure of $D_{n+1}[u]$, i.e., that it is a unit in every valuation overring $W$ of $D_{n+1}[u]$ : If the center of $W$ on $D_{n+1}$ is the same as that of $V_{n+1}$, then $W$ contains the localization $V_{n+1}$ of $D_{n+1}$ at that center, and so $b$ is a unit in $W$. Otherwise $r$ is a unit in $W$, so $R_{n+1}[u] \subseteq W$, giving the same result.

EXAMPLE 3.2. A principal-plosive GCD-domain that is the intersection of a locally finite family of rank two valuation domains and has a 
localization that is not plosive: Let $k$ denote a countable algebraically closed field and $x$ and $y$ be indeterminates. Let $h_{1}, h_{2}, \ldots$ be the nonassociate irreducible polynomials in $k[x, y]$, and let $q_{1}, q_{2}, \ldots$ be the subsequence of $h_{1}, h_{2}, \ldots$ for which $q_{i}(0,0)=0$. (Thus, the $h_{i}$-or rather $h_{i}=0$-are the irreducible curves in the plane $k^{2}$, and the $q_{i}$ are the ones through the origin.) For each positive integer $i$, pick a point $z_{i}=\left(x_{i}, y_{i}\right)$ and a linear polynomial $l_{i}$ for which:

(1) $z_{i} \neq(0,0)$;

(2) $z_{i}$ is a regular point on $q_{i}$, i.e., $q_{i}\left(z_{i}\right)=0$ and the localization of $k[x, y] /\left(q_{i}\right)$ at the maximal ideal $\left(x-x_{i}, y-y_{i}\right) /\left(q_{i}\right)$ is a discrete rank one valuation domain;

(3) $h_{j}\left(z_{i}\right) \neq 0$ for all $j<i$;

(4) $l_{j}\left(z_{i}\right) \neq 0$ for all $j<i$;

(5) $l_{i}\left(z_{i}\right)=0$;

(6) $l_{i}(0,0) \neq 0$;

(7) $l_{i}\left(z_{j}\right) \neq 0$ for all $j<i$; and

(8) $l_{i}$ is not tangent to $q_{i}$ at $z_{i}$.

(Thus, there is one $z$ for each curve $q$, no more than a finite number on each curve $h$, and the lines $l$ hit only one $z$ and not the origin, and the local ring of the plane $k^{2}$ at $z_{i}$ is regular, with regular parameters $q_{i}, l_{i}$.) Let $W_{i}$ denote the localization of $k[x, y]$ at the prime $\left(q_{i}\right)$, and let $V_{i}$ be the inverse image in $W_{i}$ of the valuation domain at $z_{i}$ on the curve $q_{i}$ from (2). Thus, $V_{i}$ is a rank two valuation domain.

Set $D=\bigcap_{i} V_{i}$, and let $Q_{i}$ and $P_{i}$ denote the contractions to $D$ of the height one and height two primes of $V_{i}$, respectively. Note that a rational function $f$ is in $P_{i}$ if and only if the denominator of $f$ has no component through the origin and the restriction of $f$ to the curve $q_{i}$ has positive value at $z_{i}$, i.e., is in the maximal ideal of the valuation domain of (2). Since $k[x, y] \subseteq V_{i} \subseteq W_{i}, k[x, y] \subseteq D \subseteq$ $\bigcap_{i} W_{i}=k[x, y]_{(x, y)}$. Since no $h_{i}$ contains infinitely many $z_{j}$, the family $\left\{V_{i}\right\}$ is locally finite. Let $f \in P_{i}$. Since $l_{i}$ has value 1 at $z_{i}$ and neither the origin nor any other $z_{j}$ is on $l_{i}, f / l_{i} \in V_{j}$ for all $j$, i.e., $f / l_{i} \in D$. Thus, $P_{i}=l_{i} D$. Moreover, for $j \neq i$, let $n$ be the value at $z_{i}$ of the restriction of the function $q_{j}$ to the curve $q_{i}$; then $q_{j} / l_{i}^{n}$ is an element of $Q_{j}$ not in $P_{i}$. Thus, $V_{i}$ is the localization of $D$ at $P_{i}$. By Proposition 2.5, $D$ is principal-plosive. But $k[x, y]_{(x, y)}$ is a localization of $D$ that is not plosive.

This example shows that the one-dimensional hypothesis in [H1, Proposition 1.2] is necessary, despite a remark to the contrary in [GW]. 


\section{REFERENCES}

[An] D. D. Anderson, Star-operations induced by overrings, Comm. Algebra, 16 (1988), 2535-2553.

[AA] D. D. Anderson and D. F. Anderson, Multiplicatively closed subsets of fields, Houston J. Math., 13 (1987), 1-11.

[AM] M. Atiyah and I. MacDonald, Introduction to Commutative Algebra, Addison-Wesley, Reading, Massachusetts, 1969.

[Au] K. Aubert, Divisors of finite character, Ann. Math. Pura Appl., 133 (1983), 327-361.

[B] N. Bourbaki, Commutative Algebra, Hermann, Paris, and Addison-Wesley, Reading, MA, 1972.

[BR] J. Brewer and E. Rutter, $D+M$ constructions with general overrings, Michigan Math. J., 23 (1976), 33-41.

[Da] E. Davis, Overrings of commutative rings II. Integrally closed overrings, Trans. Amer. Math. Soc., 110 (1964), 196-212.

[Do] D. Dobbs, A characterization of QR-domains, C. R. Acad. Sci. Canada, to appear.

[Gi1] R. Gilmer, The pseudo-radical of a commutative ring, Pacific J. Math., 19 (1966), 275-284.

[Gi2] _ Multiplicative Ideal Theory, Marcel Dekker, New York/London, 1972.

[GiO] R. Gilmer and J. Ohm, Integral domains with quotient overrings, Math. Ann., 153 (1964), 97-103.

[Go] O. Goldman, On a special class of Dedekind domains, Topology (Suppl. 1), 3 (1964), 113-118.

[GW] A. Grams and H. Warner, Irreducible divisors in domains of finite character, Duke Math. J., 42 (1975), 271-284.

[Gr1] M. Griffin, Some results on Prüfer v-multiplication rings, Canad. J. Math., 19 (1967), 710-722.

[Gr2] _ Rings of Krull type, J. Reine Angew. Math., 229 (1968), 1-27.

[HH] J. Hedstrom and E. Houston, Some remarks on star-operations, J. Pure Appl. Algebra, 18 (1980), 37-44.

[H1] W. Heinzer, Quotient overrings of integral domains, Mathematika, 17 (1970), 139-148.

[H2] _ An essential integral domain with a non-essential localization, Canad. J. Math., 33 (1981), 400-403.

[HO1] W. Heinzer and J. Ohm, Defining families for integral domains of real finite character, Canad. J. Math., 24 (1972), 1170-1177.

[HO2] _ Noetherian intersections of integral domains, Trans. Amer. Math. Soc., 167 (1972), 291-308.

[HO3] _ An essential domain that is not a v-multiplication ring, Canad. J. Math., 25 (1973), 856-861.

[J] P. Jaffard, Les Systèmes d'Idéaux, Dunod, Paris, 1960.

[K] I. Kaplansky, Commutative Rings, Univ. of Chicago Press, Chicago, 1974.

[LM] M. Larsen and P. McCarthy, Multiplicative Theory of Ideals, Academic Press, New York/London, 1971.

[M] H. Matsumura, Commutative Ring Theory, Cambridge University Press, Cambridge, England, 1986.

[MZ] J. Mott and M. Zafrullah, Unruly Hilbert domains, Canad. Math. Bull., 33 (1990), 106-109. 
[N] M. Nagata, Local Rings, Interscience, New York/London/Sydney, 1962.

[P] R. Pendleton, $A$ characterization of Q-domains, Bull. Amer. Math. Soc., 72 (1966), 499-500.

[ZS] O. Zariski and P. Samuel, Commutative Algebra, vol. II, Van Nostrand, Princeton, N.J., 1960.

Received September 2, 1988. The first author gratefully acknowledges the support of NSF Grant DMS- 8800762 and the second author the hospitality of Purdue University while this work was done.

Purdue University

West LafayetTe, IN 47907

AND

Colgate University

HAMILTON, NY 13346 


\title{
PACIFIC JOURNAL OF MATHEMATICS EDITORS
}

\author{
V. S. VARADARAJAN \\ (Managing Editor) \\ University of California \\ Los Angeles, CA 90024-1555-05 \\ Herbert Clemens \\ University of Utah \\ Salt Lake City, UT 84112 \\ ThOMAS ENRIGHT \\ University of California, San Diego \\ La Jolla, CA 92093
}

R. FINN

Stanford University

Stanford, CA 94305

HeRmanN FlaschKa

University of Arizona

Tucson, AZ 85721

VAUGHaN F. R. Jones

University of California

Berkeley, CA 94720

Steven Kerckhoff

Stanford University

Stanford, CA 94305

\section{C. MOORE}

University of California

Berkeley, CA 94720

MaRTin SCHARLEMANN

University of California

Santa Barbara, CA 93106

HAROLD STARK

University of California, San Diego

La Jolla, CA 92093

\section{ASSOCIATE EDITORS}
R. ARENS
E. F. BECKENBACH (1906-1982)
B. H. NeumanN
F. WOLF
(1904-1989)
K. YosHidA

\section{SUPPORTING INSTITUTIONS}

UNIVERSITY OF ARIZONA
UNIVERSITY OF BRITISH COLUMBIA
CALIFORNIA INSTITUTE OF TECHNOLOGY
UNIVERSITY OF CALIFORNIA
MONTANA STATE UNIVERSITY
UNIVERSITY OF NEVADA, RENO
NEW MEXICO STATE UNIVERSITY
OREGON STATE UNIVERSITY
UNIVERSITY OF ARIZONA
UNIVERSITY OF BRITISH COLUMBIA UNIVERSITY OF CALIFORNIA
MONTANA STATE UNIVERSITY
NEW MEXICO STATE UNIVERSITY

\author{
UNIVERSITY OF SOUTHERN CALIFORNIA \\ STANFORD UNIVERSITY \\ UNIVERSITY OF HAWAII \\ UNIVERSITY OF TOKYO \\ UNIVERSITY OF UTAH \\ WASHINGTON STATE UNIVERSITY \\ UNIVERSITY OF WASHINGTON
}
UNIVERSITY OF OREGON

The Supporting Institutions listed above contribute to the cost of publication of this Journal, but they are not owners or publishers and have no responsibility for its content or policies.

\begin{abstract}
Mathematical papers intended for publication in the Pacific Journal of Mathematics should be in typed form or offset-reproduced (not dittoed), double spaced with large margins. Please do not use built up fractions in the text of the manuscript. However, you may use them in the displayed equations. Underline Greek letters in red, German in green, and script in blue. The first paragraph must be capable of being used separately as a synopsis of the entire paper. In particular it should contain no bibliographic references. Please propose a heading for the odd numbered pages of less than 35 characters. Manuscripts, in triplicate, may be sent to any one of the editors. Please classify according to the 1980 Mathematics Subject Classification (1985 Revision) scheme which can be found in the December index volumes of Mathematical Reviews. Supply name and address of author to whom proofs should be sent. All other communications should be addressed to the managing editor, or Elaine Barth, University of California, Los Angeles, California 90024-1555-05.

There are page-charges associated with articles appearing in the Pacific Journal of Mathematics. These charges are expected to be paid by the author's University, Government Agency or Company. If the author or authors do not have access to such Institutional support these charges are waived. Single authors will receive 50 free reprints; joint authors will receive a total of 100 free reprints. Additional copies may be obtained at cost in multiples of 50 .
\end{abstract}

The Pacific Journal of Mathematics (ISSN 0030-8730) is published monthly. Regular subscription rate: $\$ 190.00$ a year (12 issues). Special rate: $\$ 95.00$ a year to individual members of supporting institutions.

Subscriptions, orders for numbers issued in the last three calendar years, and changes of address should be sent to Pacific Journal of Mathematics, P.O. Box 969, Carmel Valley, CA 93924, U.S.A. Old back numbers obtainable from Kraus Periodicals Co., Route 100, Millwood, NY 10546.

The Pacific Journal of Mathematics at P.O. Box 969, Carmel Valley, CA 93924 (ISSN 0030-8730) is published monthly. Second-class postage paid at Carmel Valley, California 93924, and additional mailing offices. Postmaster: send address changes to Pacific Journal of Mathematics, P.O. Box 969, Carmel Valley, CA 93924.

\section{PUBLISHED BY PACIFIC JOURNAL OF MATHEMATICS, A NON-PROFIT CORPORATION}




\section{Pacific Journal of Mathematics}

Vol. 145, No. $2 \quad$ October, 1990

Chong Hsio Fang and Minking Eie, On the values of a zeta function at

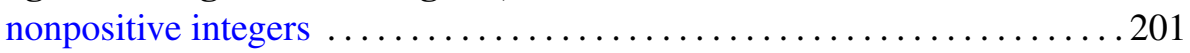

Howard D. Fegan, Brian F. Steer and L. Whiteway, Spectral symmetry of the Dirac operator for compact and noncompact symmetric pairs ..... 211

William James Heinzer and David C. Lantz, Integral domains that lose

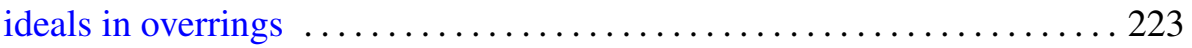

Alexander Eben Koonce, Relations among generalized characteristic classes .......................................239

M. S. Narasimhan and Günther Trautmann, Compactification of

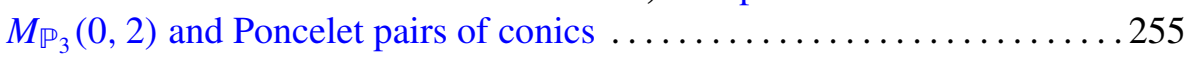

James Alexander Reeds, III and Lawrence A. Shepp, Optimal paths for a car that goes both forwards and backwards . ................. 367

Ai-Nung Wang, Constant mean curvature surfaces on a strip ............ 395 